This article is licensed under the Creative Commons Attribution-NonCommercial-NoDerivatives 4.0 International License (CC BY-NC-ND) (http://www.karger.com/Services/OpenAccessLicense). Usage and distribu-

tion for commercial purposes as well as any distribution of modified material requires written permission.

\title{
Acknowledgements to Reviewers
}

The Editor-in-Chief and Editorial Board members wish to express their wholehearted appreciation to the below-listed reviewers of Liver Cancer for their critical evaluation and competent comments on the manuscripts submitted to the journal in the year 2020 (January 1, 2020 to November 9, 2020). We had constructive suggestions from the referees aimed to improve the quality of the manuscripts for publication that were both of interest to our readers and of high and acceptable standards. It is our hope that your invaluable contribution to the journal will continue for the benefit of our readers in the years to come. Many thanks.

Ghassan Abou-Alfa, New York, NY, USA

Hiroshi Aikata, Hiroshima, Japan

Mideia Akritidou, Athens, Greece

Yasuaki Arai, Tokyo, Japan

Junichi Arita, Tokyo, Japan

Yasuhiro Asahina, Tokyo, Japan

Ahmed Ba-Ssalamah, Vienna, Austria

Sun Hyun Bae, Bucheon, Korea

K. Arnold Chan, Taipei, Taiwan

Stephen L. Chan, Shatin, Hong Kong, China

Alex Hongsheng Chang, Shanghai, China

Jason Chia-Hsien Cheng, Taipei, Taiwan

Andrea Piero Chierici, Vimercate, Italy

Joon-Il Choi, Seoul, Korea

Su Pin Choo, Singapore, Singapore

Christian Cotsoglou, Vimercate, Italy

Amanda Jean Craig, Bethesda, MD, USA

Alessandro Cucchetti, Forlì, Italy

Jose Debes, Minneapolis, MN, USA

Julien Edeline, Rennes, France

Susumu Eguchi, Nagasaki, Japan

Hirayuki Enomoto, Nishinomiya, Japan

Yuman Fong, Duarte, CA, USA

Junji Furuse, Mitaka, Japan

Mihnea-Alexandru Găman, Bucharest, Romania

Giovanni Galati, Rome, Italy

Peter Robert Galle, Mainz, Germany

Satoshi Goshima, Hamamatsu, Japan

Grace L. Guo, Piscataway, NJ, USA

Satoru Hagiwara, Osaka, Japan

Kiyoshi Hasegawa, Tokyo, Japan

Takeshi Hatanaka, Maebashi, Japan

Etsuro Hatano, Nishinomiya, Japan

Hikita Hayato, Osaka, Japan

Keisuke Hino, Kurashiki, Japan

Atsushi Hiraoka, Matsuyama, Japan

Masashi Hirooka, Ehime, Japan

Ming-Chih Ho, Taipei, Taiwan

Masao Honda, Kanazawa, Japan

Masatoshi Hori, Kobe, Japan

Sen-Yung Hsieh, Taoyuan, Taiwan
Chiun Hsu, Taipei, Taiwan

Chih-Hung Hsu, Taipei, Taiwan

Yi-Hsiang Huang, Taipei, Taiwan

Shin Hwang, Seoul, Korea

Masafumi Ikeda, Kashiwa, Japan

Yoshitaka Inaba, Nagoya, Japan

Woo Kyoung Jeong, Seoul, Korea

Philip J. Johnson, Liverpool, UK

Ijin Joo, Seoul, Korea

Toshimi Kaido, Tokyo, Japan

Tatsuo Kanda, Tokyo, Japan

Hyun-Cheol Kang, Seoul, Korea

Hyo-Jin Kang, Seoul, Korea

Jia-Horng Kao, Taipei, Taiwan

Kazuya Kariyama, Okayama, Japan

Takumi Kawaguchi, Kurume, Japan

Yoshikuni Kawaguchi, Tokyo, Japan

Yusuke Kawamura, Tokyo, Japan

Tomokazu Kawaoka, Hiroshima, Japan

Young Jun Kim, Seoul, Korea

Jin Hyoung Kim, Seoul, Korea

Tae-Hyung Kim, Seoul, Korea

Tomoki Kimura, Hiroshima, Japan

Ryuichi Kita, Osaka, Japan

Norihiro Kokudo, Tokyo, Japan

Yuko Kono, San Diego, CA, USA

Shoji Kubo, Osaka, Japan

Masatoshi Kudo, Osaka-Sayama, Japan

Takashi Kumada, Ogaki, Japan

Yutaka Kurebayashi, Shinjuku, Japan

Masayuki Kurosaki, Tokyo, Japan

Teiji Kuzuya, Fujita, Japan

Jae Young Lee, Seoul, Korea

Jooho Lee, Seongnam-si, Korea

Huei-Lung Liang, Kaohsiung, Taiwan

Shi-Ming Lin, Taoyuan, Taiwan

Vincenzo Mazzaferro, Milan, Italy

Yasunori Minami, Osaka-Sayama, Japan

Yoshihiro Mise, Tokyo, Japan

Shiro Miyayama, Fukui, Japan

Michihisa Moriguchi, Kyoto, Japan

\section{Karger $\stackrel{\text { L }}{ }$}


Manabu Morimoto, Yokohama City, Japan

Utaroh Motosugi, Yamanashi, Japan

Takamichi Murakami, Kobe, Japan

Hayato Nakagawa, Tokyo, Japan

Osamu Nakashima, Kurume, Japan

Nobuaki Nakayama, Saitama, Japan

Masashi Ninomiya, Tohoku, Japan

Naoshi Nishida, Osaka-Sayama, Japan

Hiroki Nishikawa, Osaka, Japan

Karim-Maximilian Niyazi, Munich, Germany

Kazuhiro Nouso, Okayama, Japan

Kazushi Numata, Yokohama, Japan

Sadahisa Ogasawara, Chiba, Japan

Chikara Ogawa, Takamatsu, Japan

Toshiyuki Okumura, Tsukuba, Japan

Takuji Okusaka, Tokyo, Japan

Joong-Won Park, Goyang, Korea

Mikin Patel, Tucson, AZ, USA

Markus Peck-Radosavljevic,

Klagenfurt am Wörthersee, Austria

Matthias Pinter, Vienna, Austria

Fabio Piscaglia, Bologna, Italy

Timothy L. Pruett, Minneapolis, MN, USA

Maria Reig, Barcelona, Spain

Hyunchul Rhim, Seoul, Korea

Lorenza Rimassa, Milan, Italy

Lewis R. Roberts, Rochester, MN, USA

Stephanie Rössler, Heidelberg, Germany

Issei Saeki, Ube, Japan

Naoya Sakamoto, Hokkaido, Japan

Yoshihiro Sakamoto, Tokyo, Japan

Michiie Sakamoto, Tokyo, Japan

Dominik Schramm, Halle, Germany

Masataka Seike, Oita, Japan

Olivier Seror, Bondy, France

Ying-Chun Shen, Taipei, Taiwan

Shuichiro Shiina, Tokyo, Japan

Masahito Shimizu, Gifu, Japan
Makoto Shiraki, Gifu, Japan

Mitsuo Simada, Tokushima, Japan

Katsutoshi Sugimoto, Tokyo, Japan

Toshifumi Tada, Ogaki, Japan

Atsuya Takeda, Tokyo, Japan

Shing Cheng Tan, Cheras, Malaysia

Shinji Tanaka, Hiroshima, Japan

Masatoshi Tanaka, Miyama, Japan

Yasuhito Tanaka, Kumamoto, Japan

Ryosuke Tateishi, Tokyo, Japan

Kojiro Taura, Kyoto, Japan

Takeshi Terashima, Kanazawa, Japan

Han Chong Toh, Singapore, Singapore

Katsutoshi Tokushige, Tokyo, Japan

Tetsu Tomonari, Tokushima, Japan

Guido Torzilli, Rozzano (Milan), Italy

Hidenori Toyoda, Ogaki, Japan

Kaoru Tsuchiya, Tokyo, Japan

Masakatsu Tsurusaki, Osaka-Sayama, Japan

Kazuomi Ueshima, Osaka-Sayama, Japan

Maria Varela, Oviedo, Spain

Jean-Nicolas Vauthey, Houston, TX, USA

Yoshiyuki Wada, Fukuoka, Japan

Tmohiro Watanabe, Osaka-Sayama, Japan

Chun-Ying Wu, Taipei, Taiwan

Hui-Xiong Xu, Shanghai, China

Takahiro Yamasaki, Ube, Japan

Taro Yamashita, Kanazawa, Japan

Tatsuya Yamashita, Kanazawa, Japan

Ju Yang, Los Angeles, CA, USA

Tian Yang, Shanghai, China

Hirohisa Yano, Kurume, Japan

Hiroshi Yatsuhashi, Omura, Japan

Thomas Yau, Hong Kong, China

Masashi Yoneda, Aichi, Japan

Changhoon Yoo, Seoul, Korea

Sang Min Yoon, Seoul, Korea

Jian Zhou, Shanghai, China 